\title{
Efficient hearing screening in noise-exposed listeners using the Digit Triplet test
}

For the final published version, please refer to:

Jansen S., Luts H., Dejonckere P., van Wieringen A. \& Wouters J. 2013. Efficient hearing screening in noise-exposed listeners using the digit triplet test. Ear and hearing, 34, 773-8.

Sofie JANSEN ${ }^{1}$, Heleen LUTS ${ }^{1}$, Philippe DEJONCKERE ${ }^{1,2}$, Astrid VAN WIERINGEN $^{1}$, \& Jan WOUTERS ${ }^{1}$

${ }^{1}$ KU Leuven - University of Leuven, Department of Neurosciences, ExpORL, Leuven, Belgium

${ }^{2}$ Federal Institute of Occupational Diseases, Brussels, Belgium

\section{Key words:}

Hearing screening, noise-induced hearing loss, sensitivity and specificity

\section{Corresponding author:}

Sofie Jansen, ExpORL, KU Leuven

Herestraat 49, bus 721

B-3000 Leuven

BELGIUM

Tel: $\quad$ +3216330477

Sofie.Jansen@med.kuleuven.be 


\begin{abstract}
Objective: Hearing screening in occupational medicine is generally based on pure-tone threshold audiometry. However, reliable and valid thresholds can only be obtained in a sound-proof room, using a high-quality, well-calibrated audiometer, and by a well-trained administrator. Thresholds also need to be determined for several audiometric frequencies. This makes the test timeconsuming and expensive, which is not ideal for the screening of large populations. A speech-innoise test, by contrast, does not have the above-mentioned requirements. Because it can be implemented as a quick automated self-test, possibly over the Internet, a speech-in-noise test is highly advantageous for screening purposes. However, its sensitivity for (isolated) high-frequency hearing loss, as typically seen in noise-exposed listeners, was unclear up to present. In this study, the authors investigated the sensitivity and specificity of the Digit Triplet speech-in-noise test for detecting and monitoring (early-stage) high-frequency hearing loss, and its similarity across two different language versions. Design: 118 noise-exposed workers, representing a wide range from no to severe high-frequency hearing loss, completed the French or Dutch version of the broadband Digit Triplet self-test in an office-like room. Pure-tone thresholds, collected by a professional audiologist in favorable settings, served as the reference. Results: The 84 Dutch-speaking participants showed a very strong linear relation between the reference and the Digit Triplet test, with the pure-tone average at $2,3,4$, and $6 \mathrm{kHz}$ as a strong predictor $(\mathrm{R}=0.86)$ for the speech reception threshold. The sensitivity and specificity to detect mild high-frequency hearing loss were $92 \%(61 / 66)$ and $89 \%(16 / 18)$, respectively. The area under the receiver operating characteristic $(\mathrm{ROC})$ curve was very high $(\geq 0.91)$ for several degrees of high-frequency hearing loss. With a withinsubject standard deviation of only $0.8 \mathrm{~dB}$, the Digit Triplet test also had a low measurement error. The results of the 34 French-speaking subjects showed a highly similar trend. Conclusions: The Digit Triplet test proves to have a high sensitivity and specificity for detecting different degrees of highfrequency hearing loss. Given its ease of use, this test is very suitable for screening purposes in occupational medicine, and potentially for the screening of adolescents at risk of a recreational noise-induced hearing loss.
\end{abstract}

\title{
Introduction
}

A vital part of a hearing conservation program for noise-exposed workers, is the early detection of noise-induced hearing loss and monitoring of further deterioration by means of periodic hearing screening in all these employees. Generally, occupational hearing screening is based on classical pure-tone threshold audiometry, where threshold shifts in the high frequencies around 4 and $6 \mathrm{kHz}$ are a sensitive indicator of (early-stage) damage due to noise exposure (Dempsey, 1985; Rösler, 1994; Nelson et al, 2005; Seixas et al, 2012; Marlenga et al, 2012). However, threshold audiometry has some (technical) requirements which can be difficult to fulfill in a screening setting, such as the availability of a sound-proof room, a well-calibrated audiometer, a qualified test administrator, and a sufficient amount of time to determine thresholds at several audiometric frequencies. In case one or more of these requirements cannot be achieved, there is a high chance of getting unreliable and 
invalid test outcomes, as reported in literature (Dobie, 1983; Halloran et al, 2009; Dejonckere et al, 2010).

A speech-in-noise test, in which supra-threshold stimuli are presented in a competing background noise, might provide a solution for the above-mentioned problems. First of all, there is no need to conduct the test in a sound-proof booth. A quiet office-like room, where the ambient noise will have a lower masking effect than the presented background noise, will thus be sufficient. Secondly, changes of 5 to $10 \mathrm{~dB}$ to the absolute presentation level of the test do not influence a listener's speech reception threshold (SRT), which is determined relatively to the noise level, in dB SNR (signalto-noise ratio) (Plomp \& Mimpen, 1979b). Third, the use of domestic audio equipment (standard computer sound card and headphones) has also been shown to be adequate for testing speech recognition in noise (Culling et al, 2005). Fourth, the test can easily be implemented as a fully automated PC-based self-test, so that no test administrator is needed (Smits et al, 2004; Smits \& Houtgast, 2005). And fifth, the SRT can adaptively be determined in 3 to 4 minutes. All these technical advantages make a speech-in-noise test very suitable for screening purposes.

The relationship between the SRT and pure-tone thresholds in noise-exposed workers has previously been studied by Smoorenburg and colleagues (Smoorenburg et al, 1982; Smoorenburg, 1992; Bosman \& Smoorenburg, 1995), who used lists of 13 everyday sentences presented in stationary speech-shaped noise, to determine the SRT (Plomp \& Mimpen, 1979a). The thresholds at the higher frequencies ( 2 and $4 \mathrm{kHz}$ ) were found to be the strongest predictors for the SRT. However, they could still only explain $52 \%$ of the variance $(R=0.72)$. Thresholds for all frequencies together could also only explain 57\% (Smoorenburg, 1992). Four possible reasons for this rather low correlation can be put forward. First, non-auditory cognitive aspects, such as working memory or linguistic skills, are considered to be likely to influence a listener's SRT, especially when using more complex sentences (Akeroyd, 2008). A second reason might be the measurement error when estimating a listener's SRT using one list of 13 sentences. This measurement error or 'test-retest reliability', which can be quantified as the quadratic mean of the within-subject standard deviations for repeated measurements, lies in the order of 1.0 to $1.2 \mathrm{~dB}$ (Plomp \& Mimpen, 1979a; Nilsson et al, 1994). Considering the small range of test outcomes (roughly from -5 to $+15 \mathrm{~dB} S N R$ ), this measurement error is relatively high. A third disturbing factor is the non-linearity of the relation between the SRT and pure-tone thresholds within the group of listeners with a mild hearing loss: Although having a slight pure-tone detection loss of 10 to $15 \mathrm{~dB} \mathrm{HL}$, these subjects do not yet show any loss in SRT (Smoorenburg, 1992). The high contextual redundancy in everyday sentences, enabling top-down processing, might explain this insensitivity of the SRT for very small hearing losses. And fourth, the two tests measure different auditory abilities (detecting pure tones versus discriminating and identifying complex speech stimuli embedded in competing noise). It can be assumed that a certain hearing impairment might affect both abilities differently (Shamma, 2011).

More recently, a different type of speech-in-noise test was proposed, based on simple three-digit sequences instead of everyday full sentences(Smits et al, 2004). This "Digit Triplet test" was 
implemented as an automated self-screening test administered by telephone and proved to be applicable for hearing screening in a general population (Smits et al, 2004; Smits \& Houtgast, 2005; Smits et al, 2006; Jansen et al, 2010; Vlaming et al, 2011; Watson et al, 2012). A first advantage of the Digit Triplet test is the low measurement error that was found in all these studies, with a withinsubject standard deviation of only $0.7 \mathrm{~dB}$. Furthermore, using this simplified test, the SRT can be assumed to be less influenced by non-auditory cognitive abilities. And third, digit triplets have a lower redundancy compared to meaningful sentences, and therefore might be more sensitive to the first signs of hearing loss.

The rationale for this study was to investigate the potential of the Digit Triplet test for hearing screening in a noise-exposed population, by studying its sensitivity for (isolated) high-frequency hearing loss. It is hypothesized that, compared to a sentence SRT (Smoorenburg, 1992), the Digit Triplet SRT will show a stronger linear correlation with the pure-tone thresholds, and therefore, will have a high sensitivity and specificity. Furthermore, two language versions of the Digit Triplet test are compared to examine the across-language generalization.

\section{Materials and methods}

\section{Study population and data collection}

The participants in this study were recruited from five major industrial companies across Belgium and had an average 8-hour working day noise exposure level (Lex, 8h) of at least $80 \mathrm{~dB}$ A. Three candidates were excluded because of their poor mastery of the Dutch and French language. In total 118 workers participated, of which 116 were male, aged between 22 and 59 years. They varied largely regarding the number of years working in noise, the type and level of noise to which they were exposed, and the adequacy of their hearing protection use.

All data were collected at the ENT department of the Federal Institute of Occupational Diseases. For each participant, pure-tone thresholds and the Digit Triplet SRT were measured on the same day, in randomized order. The pure-tone thresholds were measured under optimal conditions, i.e. in a sound-proof booth, by a professional audiologist. The Digit Triplet test was carried out in an officelike room and the participants completed the test independently, without a test administrator. The Digit Triplet SRT was only determined for one randomly selected ear.

\section{Measurement of the Digit Triplet SRT}

The Digit Triplet test was conducted on a PC, with sound stimuli transmitted through a 24-bit sound card to a pair of Sennheiser HDA200 headphones. The software APEX (Francart et al, 2008) was used to play back the triplets-in-noise at the desired SNR, and to automatically score the subject's typed response. For each SRT measurement, a list of 27 triplets, randomly selected from 10 available test lists, was presented using a simple up-down adaptive procedure with steps of $2 \mathrm{~dB}$. The level of the stationary speech-shaped noise was fixed at $65 \mathrm{~dB}$ SPL and the first triplet was presented at $0 \mathrm{~dB}$ SNR. The noise started $1 \mathrm{~s}$ before and stopped $500 \mathrm{~ms}$ after each triplet. All three digits needed to be 
identified correctly in order to decrease the SNR. After the response to the $27^{\text {th }}$ triplet was scored and the SNR of the (non-presented) next triplet was determined, the SRT was calculated by averaging the last 22 SNRs. The mean duration of one test was 4 min $11 \mathrm{~s}$.

From the 118 participants there were 34 French-speaking persons who completed the French Digit Triplet test. Details on the development of this test are described by Jansen et al (2010). The other 84 participants were speakers of Dutch and completed the Flemish Digit Triplet test. The Flemish version was developed analogous to the French one, and was based on the digit recordings of the Leuven Intelligibility Number Test (van Wieringen \& Wouters, 2008). The only difference with the French version is (1) the omission of the digits 7 and 9, as these are disyllabic words in Dutch, (2) the absence of the announcement words "the numbers ..." preceding each triplet, and (3) the absence of a triplet intonation. For both languages, the individual digits had been equated for intelligibility, resulting in a very steep slope of psychometric function (above 20\%/dB) (Jansen et al, 2010). Evaluation in 12 young adult normal-hearing listeners revealed a reference SRT of $-11.7 \mathrm{~dB}$ SNR (SD = $0.6 \mathrm{~dB}$ SNR) for the Flemish test. For the French version, the reference SRT was $-10.5 \mathrm{~dB}$ SNR (SD = $0.3 \mathrm{~dB}$ SNR) (Jansen et al, 2010).

\section{Results}

\section{Distribution of the pure-tone thresholds}

The distribution of the pure-tone thresholds of the 118 tested ears is shown in Figure 1 and is highly comparable to distributions for similar study samples reported in the literature (Smoorenburg, 1992; Leensen et al, 2011b). In general, participants had normal or near-normal thresholds for the lower frequencies up to $1 \mathrm{kHz}$, whereas thresholds in the high-frequency range were clearly elevated. Besides a natural deterioration of hearing thresholds due to age which might be present in the older participants, the damaging effect of noise exposure was clearly visible in the audiometric notch around 4 and $6 \mathrm{kHz}$.

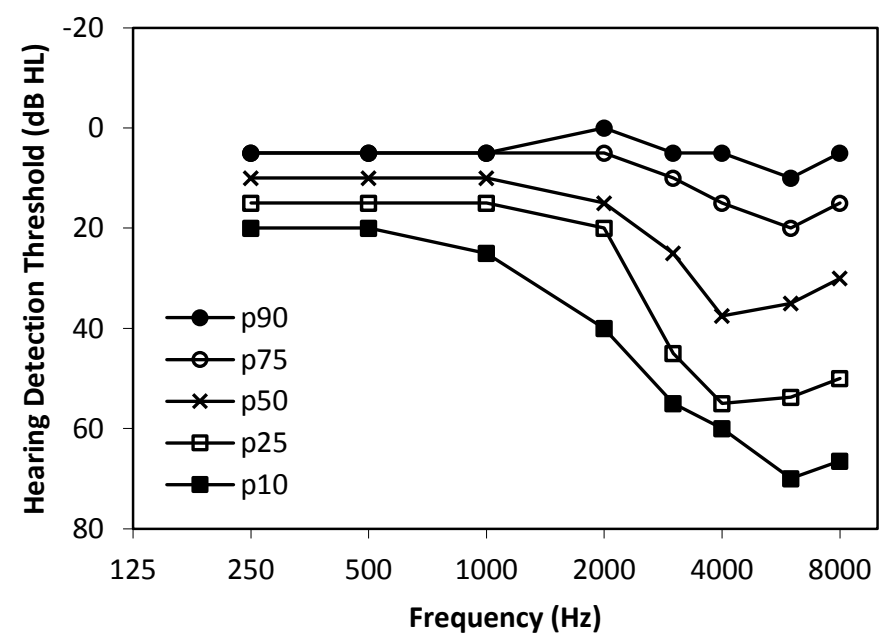

Figure 1 Distribution of the pure-tone thresholds for the 118 tested ears. Each line represents a different percentile (p10, p25, p50, p75, and p90). 
Comparing the pure-tone average (PTA) across all audiometric frequencies of the tested and nontested ears, 30 participants (25\%) turned out to have an asymmetric hearing loss (PTA difference $>5$ $\mathrm{dB})$. Due to the random selection of the test ear, 15 participants had conducted the Digit Triplet test in their better ear, and 15 subjects in their worse ear showing a PTA difference of maximally $20 \mathrm{~dB}$.

\section{Sensitivity of the Flemish Digit Triplet test}

First, the results of the larger group of subjects, i.e. the 84 Dutch-speaking participants, are described. After correcting for multicollinearity, a stepwise linear regression analysis with backward elimination was executed to examine the relation between the SRT and the pure-tone thresholds at the different audiometric frequencies. The thresholds at 2, 3, 4, and $6 \mathrm{kHz}$ contributed significantly to the prediction of the SRT. By taking the pure-tone average of these frequencies (PTA $2,3,4,6)$ as a single predictor, a strong linear correlation of 0.86 was found. Results of one listener had been excluded, for the regression analysis only, because of outlying results (absolute value of the studentized deleted residual larger than $t[1-\alpha / 2 n ; n-3]=3.574)$. A scatter plot of the SRT versus the PTA $_{2,3,4,6}$ is shown in Figure 2 (upper panel). As can be seen in this figure, the linear relation does not seem to level off at the lower end of the range (i.e. for the better listeners). This is a first indication that the Digit Triplet test might be very sensitive to detect the slightest high-frequency hearing losses.

The exact size of the measurement error of the Digit Triplet test was also determined. This measurement error can be defined as the quadratic mean of the within-subject standard deviations of repeated measurements. When only one SRT measurement is available per listener, one can compare the average SNR of the first half of the trials to the average of the second half, calculate the reliability as defined above, and then correct for the decrease in the number of trials (Smits et al, 2004). For the 84 Dutch-speaking listeners, a reliability of $0.8 \mathrm{~dB}$ on the Flemish Digit Triplet test was found.

The sensitivity of the Digit Triplet test to detect high-frequency hearing loss in a noise-exposed population could be determined for different degrees of hearing loss. The choice of a certain pass/fail criterion for the SRT should depend on the target group of the screening test and on the specific referral strategy. In this paper two potential referral schemes are discussed as an example.

A first objective to screen for could be the early warning for the first signs of high-frequency hearing loss. Based on the ISO standard on the statistical distribution of pure-tone thresholds (ISO 7029:2000), a PTA $2,3,4,6$ above $10 \mathrm{~dB} \mathrm{HL}$ can be considered as deviating (below the $10^{\text {th }}$ percentile) for a 20-year-old listener. With a cutoff SRT of -10.0 dB SNR, the Digit Triplet test yields a sensitivity of $92 \%(61 / 66)$ and a specificity of $89 \%(16 / 18)$ to detect listeners with a PTA $\mathrm{A}_{2,3,4,6}$ above $10 \mathrm{~dB} \mathrm{HL}$.

The detection of persons who are likely to benefit significantly from assistive technology such as hearing aids, can be a second screening objective. For this purpose, it is more difficult to put forward a clear-cut PTA ${ }_{2,3,4,6}$ criterion. In Belgium, a hearing aid is (partially) reimbursed when a listener's PTA $_{1,2,4}$ is $40 \mathrm{~dB}$ HL or higher. In the Dutch-speaking group, six subjects can in this way be considered 
hearing aid candidates. These are also the subjects with the highest PTA ${ }_{2,3,4,6}$ threshold (>50 dB HL). Therefore, a cutoff PTA ${ }_{2,3,4,6}$ of $50 \mathrm{~dB} \mathrm{HL}$ is considered first: when applying a cutoff SRT of $-5.8 \mathrm{~dB}$ SNR, the sensitivity and specificity equal $100 \%(7 / 7)$ and $92 \%$ (71/77), respectively. In addition, for a $40 \mathrm{~dB}$ HL criterion, a cutoff SRT of $-7.1 \mathrm{~dB}$ SNR yields a sensitivity of $88 \%(15 / 17)$ and a specificity of $87 \%(58 / 67)$.
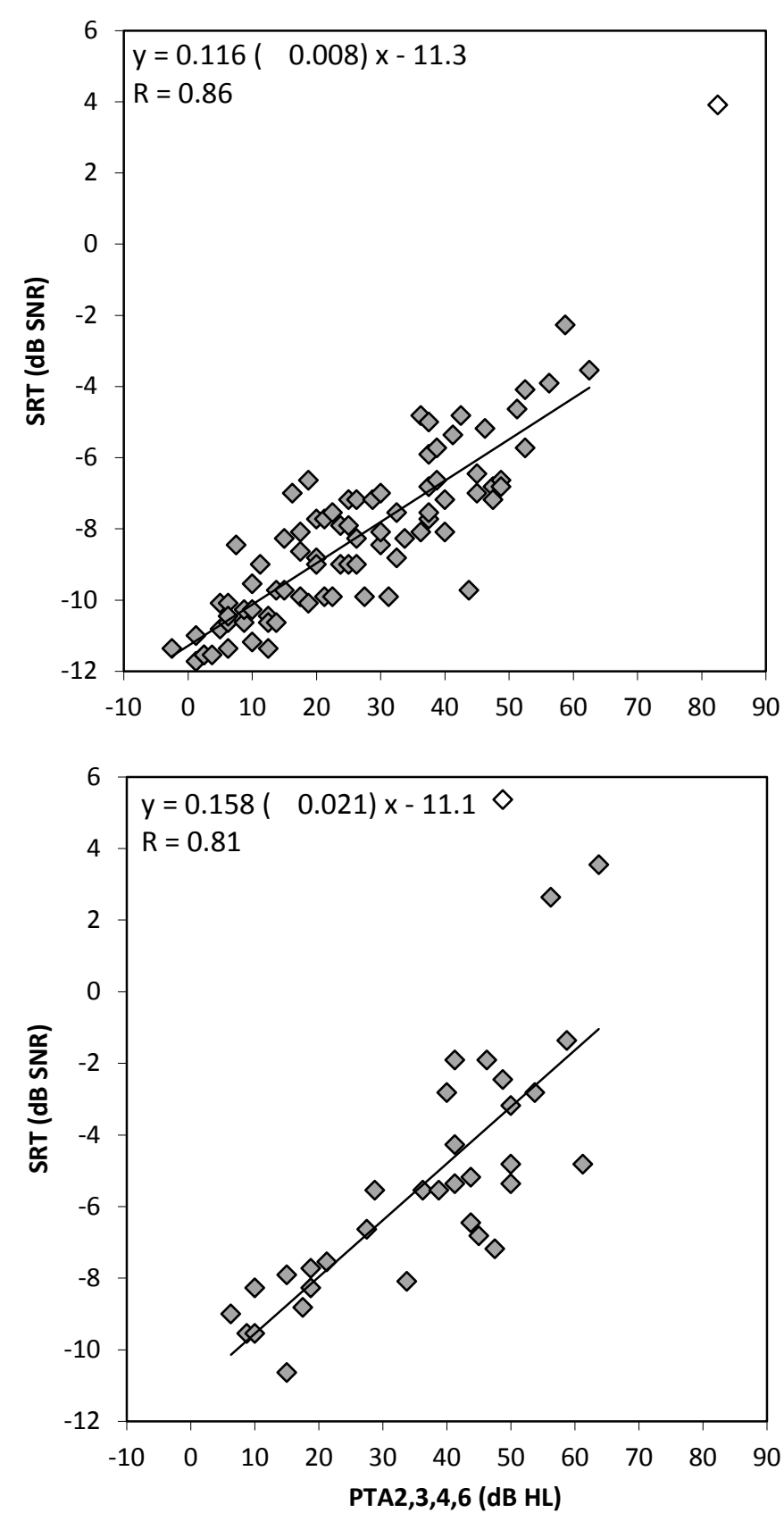

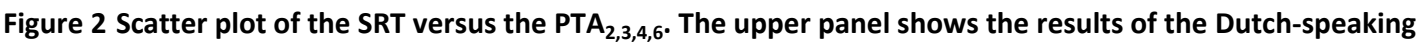
participants $(\mathrm{N}=84)$, the lower panel of the French-speaking subjects ( $N=34)$. The 2 open symbols represent outlying data points which were excluded from the linear regression model, but included in the sensitivity and specificity estimation.

In addition to these sensitivity and specificity estimates, a receiver operating characteristic (ROC) analysis was executed as well. In Table 1, the ROC area and the asymptotic $95 \%$ confidence interval is given for PTA $2,3,4,6$ cutoff values of $10,20,30,40$, and $50 \mathrm{~dB} \mathrm{HL}$. For all these cutoff values, the ROC 
area proved to be very high $(\geq 0.91)$. There was also no significant difference between the different cutoff values (all 95\% confidence intervals overlap).

\section{Across-language generalization}

Compared to the Dutch-speaking participants, similar results were found for the group of 34 Frenchspeaking subjects using the French Digit Triplet test. First, an identical test-retest reliability of $0.8 \mathrm{~dB}$ on the SRT was found. Second, the linear relation between the SRT and the PTA $A_{2,3,4,6}$ was highly comparable as well (Figure 2, lower panel). When excluding the outlying results of one listener (absolute value of the studentized deleted residual larger than $t[1-\alpha / 2 n ; n-3]=3.490$ ), a correlation coefficient of 0.81 was found.

Overall, the SRTs of the French-speaking participants tend to be somewhat higher than the SRTs of the Dutch-speaking group, corresponding to the $1.2 \mathrm{~dB}$ difference in reference SRT of the two language versions (cf. methods). When shifting the SRTs of the French-speaking participants by 1.2 $\mathrm{dB}$ and then merging the results of all 118 noise-exposed workers together, again a strong linear correlation $(R=0.84)$ and high sensitivity and specificity values were found. For the $10 \mathrm{~dB} H L$ criterion (-10.0 dB SNR), the sensitivity and specificity equaled 93\% (89/96) and 86\% (19/22). The $40 \mathrm{~dB} \mathrm{HL}$ criterion (-7.1 dB SNR) yielded a sensitivity of $86 \%$ (30/35) and specificity of $84 \%$ (70/83), and the 50 dB HL criterion (-5.8 dB SNR) resulted in a sensitivity of $92 \%(11 / 12)$ and specificity of $88 \%(93 / 106)$. Also the areas under the ROC curves for the different criteria were found to be very large $(\geq 0.92)$ for the combined results of the Dutch- and French-speaking participants (see Table 1).

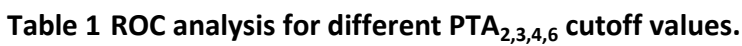

\begin{tabular}{|c|c|c|c|c|c|c|}
\hline \multirow{3}{*}{$\begin{array}{c}\text { PTA }_{2,3,4,6} \\
\text { (dB HL) }\end{array}$} & \multicolumn{3}{|c|}{ Dutch speaking only } & \multicolumn{3}{|c|}{ Dutch and French speaking } \\
\hline & \multirow{2}{*}{ area } & \multicolumn{2}{|c|}{ asymptotic $95 \% \mathrm{Cl}$} & \multirow[t]{2}{*}{ area } & \multicolumn{2}{|c|}{ asymptotic $95 \% \mathrm{Cl}$} \\
\hline & & lower bound & upper bound & & lower bound & upper bound \\
\hline 10 & 0.95 & 0.898 & 0.996 & 0.95 & 0.905 & 0.988 \\
\hline 20 & 0.91 & 0.844 & 0.970 & 0.93 & 0.889 & 0.977 \\
\hline 30 & 0.91 & 0.850 & 0.973 & 0.93 & 0.884 & 0.972 \\
\hline 40 & 0.92 & 0.849 & 0.997 & 0.92 & 0.868 & 0.972 \\
\hline 50 & 0.99 & 0.968 & 1.000 & 0.96 & 0.921 & 0.994 \\
\hline
\end{tabular}

$\mathrm{Cl}=$ confidence interval

\section{Discussion}

Supporting the findings of previous studies on the Digit Triplet test (Smits et al, 2004; Smits \& Houtgast, 2005; Jansen et al, 2010; Watson et al, 2012), these results show that reliable and quick SRTs can be obtained with this automated self-test. With a measurement error of only $0.8 \mathrm{~dB}$ and a test duration of approximately 4 minutes per ear, the test is highly suitable for hearing screening in large populations. Furthermore, current ongoing experiments prove that the test duration can be reduced to approximately 3 minutes per ear by simply enabling listeners to start giving in their response during stimulus presentation (instead of after) and by reducing the software default interval between pressing 'Enter' to play the next triplet and actually playing that triplet. 
Specifically for noise-exposed workers or others at risk of noise-induced high-frequency hearing loss, this study gives new and important insights on the sensitivity of the Digit Triplet test. As opposed to previous studies using sentence tests (Smoorenburg et al, 1982; Smoorenburg, 1992), the correlation between the Flemish Digit Triplet SRT and the PTA $\mathrm{A}_{2,3,4,6}$ was very strong $(\mathrm{R}=0.86)$ and did not seem to show a lower asymptote. As mentioned in the introduction, this stronger correlation might be explained by the lower measurement error and by a potentially lower influence of nonauditory cognitive factors on the SRT when using digit triplets compared to more complex sentences. The apparent absence of a floor effect within the group of better listeners might be explained by the reduced contextual redundancy in the speech items. Notwithstanding the fundamental difference between both tests (pure-tone detection versus supra-threshold speech identification), cochlear damage due to noise exposure seems to affect both abilities in a very similar way (Oxenham \& Bacon, 2003). As a result, very high sensitivity and specificity values were found for the Flemish Digit Triplet test, both for detecting listeners with the first signs of high-frequency hearing loss and for detecting hearing aid candidates.

The results of the French-speaking group indicate that this high sensitivity of the Flemish Digit Triplet test for high-frequency hearing loss can be generalized to other languages. Even though French and Dutch are rather different languages, the linear correlation and sensitivity and specificity remained very strong when taking together the results of both groups. Only an overall shift in SRT, based on the difference in reference values for normal-hearing listeners, needs to be applied.

Up to now, only one study in the literature reported an investigation on the sensitivity and specificity of a Digit Triplet test specifically in noise-exposed workers. Leensen et al (2011a) reported sensitivity and specificity values of $55 \%$ and $94 \%$, respectively, which is much lower than what was found in this study. Furthermore, these values were calculated based on two predefined subject groups which did not join together tightly: The 'normal-hearing listeners' had pure-tone thresholds below or equal to $15 \mathrm{~dB} \mathrm{HL}$, whereas the 'noise-exposed listeners' had one or more thresholds above $25 \mathrm{~dB} \mathrm{HL}$ in the higher frequencies, leaving a grey zone in between. The real sensitivity is thus expected to be even lower. A possible reason for these rather weak results can be found in the stimuli that were employed: Leensen and colleagues applied a bandwidth limitation ( 0.3 to $3.4 \mathrm{kHz}$ ) to the speech and noise stimuli, in order to mimic the original screening test through telephone. The higher accuracy of a broadband speech-in-noise test compared to a bandwidth limited has also been shown by Smits et al (2004), who found higher SRT-PTA correlation coefficients for sentences and triplets presented through headphones ( $R$ around 0.80 ) than for sentences and triplets presented through telephone $(R$ around 0.70 ). Although the Digit Triplet test by telephone certainly has a high value for hearing screening in a general population, with fairly good sensitivity and specificity using PTA $\mathrm{A}_{0.5,1,2}>20 \mathrm{~dB}$ $\mathrm{HL}$ as the criterion measure (Smits et al, 2004; Watson et al, 2012), the use of broadband signals and transducers is recommended when screening for the first signs of high-frequency hearing loss.

The findings of this study are also considered to be important for the rising group of adolescents frequently exposed to high sound levels during leisure activities (Dalton et al, 2001; Schmuziger et al, 
2006; Vogel et al, 2007; Torre, 2008). Recent studies show that they are at risk of a recreational noise-induced hearing loss, reporting prevalence values of approximately $15 \%$ in children and adolescents up to 19 years old (Niskar et al, 2001; Shargorodsky et al, 2010; Henderson et al, 2011). Although leisure noise may involve a more intermittent exposure than occupational noise, a similar SRT-PTA relation as described in this paper is assumed. Current sensitization and awareness campaigns aiming to reduce the prevalence of noise-induced hearing loss in adolescents aged 14 and above, are already incorporating this simple, robust, and sensitive hearing screening test on an Internet platform. The implementation of the Digit Triplet test as the official hearing screening procedure in Flemish middle and high schools is also currently under investigation.

\section{Conclusions}

This study demonstrates that a broadband Digit Triplet test is a valid and reliable alternative to puretone audiometry for detecting and monitoring hearing loss in a noise-exposed population. The test has a sensitivity and specificity of around $90 \%$, both for the identification of persons with early signs of high-frequency hearing loss and for persons who should be candidates for hearing aids. Given the ease of use of the Digit Triplet test and its relative high robustness regarding the testing environment, the audio equipment, and the output calibration, this test opens perspectives for broadly accessible hearing screening, e.g. over the Internet, for recreational and occupational hearing loss.

\section{Acknowledgements}

The authors would like to thank following occupational health physicians for recruiting the participants of this study: Johan De Pooter and Patricia Boulanger (NMBS/SNCB-holding), Ginette Van Walle (Bombardier Transportation), Anne Guyssens (Heijmans Infra NV), Rose-Mary Roussel (FN Herstal), and Jean-Michel Huberlant (ArcelorMittal Liège). The audiologists Annelies Martens, Christiane Coryn, and Reinharda Van Gool are gratefully acknowledged for administering the measurements at the Federal Institute of Occupational Diseases. 


\section{References}

Akeroyd M.A. 2008. Are individual differences in speech reception related to individual differences in cognitive ability? A survey of twenty experimental studies with normal and hearing-impaired adults. Int. J. Audiol., 47, S53-71.

Bosman A.J. \& Smoorenburg G.F. 1995. Intelligibility of Dutch CVC syllables and sentences for listeners with normal hearing and with three types of hearing impairment. Audiol. Off. organ Int. Soc. Audiol., 34, 260-84.

Culling J.F., Zhao F. \& Stephens D. 2005. The viability of speech-in-noise audiometric screening using domestic audio equipment. Int. J. Audiol., 44, 691-700.

Dalton D.S., Cruickshanks K.J., Wiley T.L., Klein B.E., Klein R., et al 2001. Association of leisure-time noise exposure and hearing loss. Audiol. Off. organ Int. Soc. Audiol., 40, 1-9.

Dejonckere P., Coryn C. \& Lebacq J. 2010. Reliability of Pure Tone Audiometry in Context of Occupational Medicine is Insufficient for an Adequate Prevention of NIHL. In 37th Neurootological Equilibriometric Soc. Congr. Bad Kissingen, Germany.

Dempsey J.J. 1985. $6000 \mathrm{~Hz}$ as an early indicator of noise-induced hearing loss. Ear Hear., 6, 159-60.

Dobie R.A. 1983. Reliability and validity of industrial audiometry: implications for hearing conservation program design. Laryngoscope, 93, 906-27.

Francart T., van Wieringen A. \& Wouters J. 2008. APEX 3: a multi-purpose test platform for auditory psychophysical experiments. J. Neurosci. Methods, 172, 283-93.

Halloran D.R., Hardin J.M. \& Wall T.C. 2009. Validity of pure-tone hearing screening at well-child visits. Arch. Pediatr. Adolesc. Med., 163, 158-63.

Henderson E., Testa M.A. \& Hartnick C. 2011. Prevalence of noise-induced hearing-threshold shifts and hearing loss among US youths. Pediatrics, 127, e39-46.

ISO 7029 2000. Acoustics - Statistical distribution of hearing thresholds as a function of age.

Jansen S., Luts H., Wagener K.C., Frachet B. \& Wouters J. 2010. The French digit triplet test: a hearing screening tool for speech intelligibility in noise. Int. J. Audiol., 49, 378-87.

Leensen M.C.J., de Laat J.A.P.M. \& Dreschler W.A. 2011a. Speech-in-noise screening tests by internet, part 1: test evaluation for noise-induced hearing loss identification. Int. J. Audiol., 50, 823-34.

Leensen M.C.J., van Duivenbooden J.C. \& Dreschler W.A. 2011b. A retrospective analysis of noiseinduced hearing loss in the Dutch construction industry. Int. Arch. Occup. Environ. Health, 84, 577-90.

Marlenga B., Berg R.L., Linneman J.G., Wood D.J., Kirkhorn S.R., et al 2012. Determinants of earlystage hearing loss among a cohort of young workers with 16-year follow-up. Occup. Environ. Med., 69, 479-84. 
Nelson D.I., Nelson R.Y., Concha-Barrientos M. \& Fingerhut M. 2005. The global burden of occupational noise-induced hearing loss. Am. J. Ind. Med., 48, 446-58.

Nilsson M., Soli S.D. \& Sullivan J.A. 1994. Development of the Hearing In Noise Test for the measurement of speech reception thresholds in quiet and in noise. J. Acoust. Soc. Am., 95, 1085-99.

Niskar A.S., Kieszak S.M., Holmes A.E., Esteban E., Rubin C., et al 2001. Estimated prevalence of noise-induced hearing threshold shifts among children 6 to 19 years of age: the Third National Health and Nutrition Examination Survey, 1988-1994, United States. Pediatrics, 108, 40-3.

Oxenham A.J. \& Bacon S.P. 2003. Cochlear compression: perceptual measures and implications for normal and impaired hearing. Ear Hear., 24, 352-66.

Plomp R. \& Mimpen A.M. 1979a. Improving the reliability of testing the speech reception threshold for sentences. Audiol. Off. organ Int. Soc. Audiol., 18, 43-52.

Plomp R. \& Mimpen A.M. 1979b. Speech-reception threshold for sentences as a function of age and noise level. J. Acoust. Soc. Am., 66, 1333-42.

Rösler G. 1994. Progression of hearing loss caused by occupational noise. Scand. Audiol., 23, 13-37.

Schmuziger N., Patscheke J. \& Probst R. 2006. Hearing in nonprofessional pop/rock musicians. Ear Hear., 27, 321-30.

Seixas N.S., Neitzel R., Stover B., Sheppard L., Feeney P., et al 2012. 10-Year prospective study of noise exposure and hearing damage among construction workers. Occup. Environ. Med., 69, 643-50.

Shamma S.A. 2011. Hearing impairments hidden in normal listeners. Proc. Natl. Acad. Sci. U. S. A., $108,16139-40$.

Shargorodsky J., Curhan S.G., Curhan G.C. \& Eavey R. 2010. Change in prevalence of hearing loss in US adolescents. JAMA J. Am. Med. Assoc., 304, 772-8.

Smits C. \& Houtgast T. 2005. Results from the Dutch speech-in-noise screening test by telephone. Ear Hear., 26, 89-95.

Smits C., Kapteyn T.S. \& Houtgast T. 2004. Development and validation of an automatic speech-innoise screening test by telephone. Int. J. Audiol., 43, 15-28.

Smits C., Kramer S.E. \& Houtgast T. 2006. Speech reception thresholds in noise and self-reported hearing disability in a general adult population. Ear Hear., 27, 538-49.

Smoorenburg G.F. 1992. Speech reception in quiet and in noisy conditions by individuals with noiseinduced hearing loss in relation to their tone audiogram. J. Acoust. Soc. Am., 91, 421-37.

Smoorenburg G.F., de Laat J.A. \& Plomp R. 1982. The effect of noise-induced hearing loss on the intelligibility of speech in noise. Scand. Audiol. Suppl., 16, 123-33. 
Torre P. 2008. Young adults' use and output level settings of personal music systems. Ear Hear., 29, 791-9.

Van Wieringen A. \& Wouters J. 2008. LIST and LINT: sentences and numbers for quantifying speech understanding in severely impaired listeners for Flanders and the Netherlands. Int. J. Audiol., $47,348-55$.

Vlaming M.S.M.G., Kollmeier B., Dreschler W.A., Martin R., Wouters J., et al 2011. HearCom: Hearing in the Communication Society. Acta Acust. united with Acust., 97, 175-92.

Vogel I., Brug J., van der Ploeg C.P.B. \& Raat H. 2007. Young people's exposure to loud music: a summary of the literature. Am. J. Prev. Med., 33, 124-33.

Watson C.S., Kidd G.R., Miller J.D., Smits C. \& Humes L.E. 2012. Telephone screening tests for functionally impaired hearing: current use in seven countries and development of a US version. J. Am. Acad. Audiol., 23, 757-67. 